\title{
Asphaltene Adsorption on Quartz Sand in the Presence of Pre-
}

\section{adsorbed Water}

\author{
Valeska Gonzalez, Spencer E. Taylor* \\ Centre for Petroleum and Surface Chemistry, Department of Chemistry, University of Surrey, \\ Guildford, Surrey GU2 7XH, UK
}

\begin{abstract}
Background and hypothesis. In the oil industry, asphaltenes are known for their tendency to aggregate in solution and to deposit on surfaces, with both properties being connected with operational problems associated with recovery, production and refining. Interactions involving asphaltenes and other crude oil components have been a major aspect of investigation in attempting to understand the full nature of these problems. Water is implicated in asphaltene behaviour in solution, for example, where it has been found to delay the deposition of asphaltene aggregates. At interfaces, there is evidence that water-in-crude oil emulsion stability is enhanced through asphaltene-water interactions, and there have been a few reports that asphaltene adsorption on mineral surfaces is reduced in the presence of water. We consider the latter aspect, because the instances of reduced adsorption to date have not attempted to quantify the effect. Previous studies showed that vapor-phase adsorption of organic molecules is reduced in the presence of pre-adsorbed water and we were interested to determine whether the same is true for liquid-phase adsorption of asphaltenes.
\end{abstract}

Experiments. The surface of quartz sand was controlled by pre-adsorption of water from different relative humidity $(\mathrm{RH})$ environments as the water adsorption isotherm is known from previous studies. These pre-conditioned sand samples were used as substrates for the 
adsorption from toluene solutions of $n$-heptane-precipitated asphaltenes (C7A) from an Athabasca oil sands bitumen. To supplement the adsorption behavior, atomic force microscopy (AFM) images were taken of the resultant sand grains at 0 and $80 \% \mathrm{RH}$, and low-field NMR was used to estimate the sand wettability.

Findings. Asphaltene adsorption on sand is sensitive to surface-adsorbed water, with $\sim 4$-fold reduction in adsorption when increasing the $\mathrm{RH}$ from 0 to $100 \%$. This is in general agreement with previous vapor-phase adsorption of small organic molecules, and is therefore believed to be the first demonstration of the effect of pre-adsorbed water on adsorption from solution. Asphaltene adsorption as a function of RH is the converse of literature water adsorption isotherm data. Three asphaltene adsorption regions have been tentatively identified based on water adsorption behavior and the literature interpretation of water structure on quartz: the highest asphaltene adsorption occurs at very low $\mathrm{RH}$, decreasing to a near-constant value in the range $\sim 40-80 \% \mathrm{RH}$, followed by a rapid decrease beyond $80 \% \mathrm{RH}$. Further analysis strongly suggests that asphaltene adsorption decreases linearly with the thickness of the adsorbed water film. The effects of RH on asphaltene adsorption are also reflected in AFM images and NMR wettability results.

Keywords: Adsorption isotherms; AFM; asphaltene; oil sand; NMR; quartz sand; relative humidity 


\section{Introduction}

Asphaltenes are separated from crude and residual oils by precipitation with alkane solvents. They are a heterogeneous class of molecules which contrast sharply in polarity and structure from the majority of the crude oil components, which accounts for their predisposition to self-aggregation and adsorption at surfaces. In practice, this leads to undesirable consequences, amongst which are deposition and wettability modification during crude oil extraction, production and refining.

A typical chemical analysis of a petroleum asphaltene is $\sim 80 \% \mathrm{C}, \sim 8 \% \mathrm{H}$ and $\sim 12 \%$ heteroatoms $(\mathrm{O}, \mathrm{N}$ and $\mathrm{S})$, with minor contributions from coordinated heavy metals. The asphaltene skeleton consists of a planar polycyclic aromatic 'nucleus' with varying degrees of aliphatic and alicyclic hydrocarbon substitution. The heteroatoms are variously distributed throughout the structure depending on the crude oil source and provide an intrinsic degree of surface activity.

Although asphaltenes are not surface-active to the same extent as classical surfactants, they do share the properties of self-aggregation and adsorption at different surfaces, including water [1].

Since water is known to be an integral part of all crude oil reservoirs, it cannot be ignored when considering surface and colloid chemical aspects of crude oil recovery and processing. Workers in this field will be particularly familiar with the presence of water as a discrete phase in water-in-crude oil emulsions, owing to the necessity to dehydrate the oil before it can be exported or refined. For this reason, systems containing water dispersed as microscopic droplets in crude oil are now relatively well understood, and adsorption of asphaltenes at the oil/water interface is considered to be an important emulsion stabilization mechanism, since despite only a modest reduction in oil/water interfacial tension, strong 
interfacial films develop over time [2-4]. It is possible that water plays an active role during asphaltene stabilization of macroscopic w/o interfaces, promoting the formation of asphaltene networks [5]. Irreversibly-adsorbed films of this type produce viscoelastic barriers which enhance the kinetic stability of water-in-crude oil emulsions [6] and are seen at aqueous interfaces of retracted pendant drops of crude oil [7] and asphaltene solutions in toluene $[6,8]$.

Self-aggregation, whether at interfaces or in solution, is understood to result from $\pi-\pi$ stacking of the asphaltene polycyclic aromatic regions [9], although electrostatic and $\mathrm{H}$ bonding contributions involving water have also been identified in model asphaltene systems [10-12]. This latter view supports other results from simulation and experiment, that low water concentrations promote asphaltene nanoaggregation in toluene solution [13], which is suggested to involve intermolecular H-bonded water bridges and asphaltene heteroatoms, e.g. $\left[\mathrm{A}_{1} \mathrm{O} \cdots \mathrm{H}-\mathrm{O}-\mathrm{H} \cdots \mathrm{NA}_{2}\right]$ or similar (the subscripts denoting different asphaltene molecules).

Likewise, solubilization of water in toluene increases in the presence of dissolved asphaltenes $[14,15]$. However, the counterintuitive finding that the adsorption capacity decreases with increasing asphaltene concentration has been explained by Andersen and coworkers as indicative of water binding to polar sites on the exterior of asphaltene nanoaggregates [15].

From the foregoing it appears that there are a number of examples where asphaltene/water interactions are implicated in the behavior of crude oils, and for which further understanding at the molecular or nano-scale is required. The present work considers a particular example of this, namely the effect of water on asphaltene adsorption on quartz sand. Although our interest stemmed originally from the structure of oil sands, it will be relevant to sandstone reservoirs in general. In the case of oil sands, however, the structure has long been thought of 
as consisting of bitumen separated from the sand surface by a thin (few nm) layer of water [16-19].

In such systems, it is important to understand how the presence of water might affect the adsorption behavior of crude oil components, such as asphaltenes. It is generally considered $[20,21]$ that deposition of asphaltenes on mineral surfaces is an important mechanism to explain wettability changes occurring in the reservoir [22-24], with the most polar asphaltene fractions showing the greatest effects [25]. This is significant, since understanding the factors that affect reservoir wettability, including the effect of water, should aid efforts to improve crude oil recovery.

Asphaltenes adsorb at solid surfaces [1], essentially to produce monolayers of nanoaggregates, the latter comprising stacks of $\sim 6$ asphaltene molecules, according to the Yen-Mullins aggregation model [26]; further aggregation of nanoaggregates to form clusters at sufficiently high concentrations leads to thicker adsorbed layers [27,28]. According to Adams' comprehensive review on asphaltene adsorption, by assuming $3 \mathrm{~nm}$ asphaltene (nano)aggregates with a density of $1200 \mathrm{~kg} / \mathrm{m}^{3}$, the maximum surface coverage for an asphaltene monolayer is calculated to be $\sim 3.6 \mathrm{mg} / \mathrm{m}^{2}$ [1]. The same review included a brief discussion of the effects of water on asphaltene adsorption on mineral surfaces, with the conclusion that water reduced adsorption due to competition for surface sites.

Here, we focus on this aspect in particular. Collins and Melrose were the first to question whether the presence of water on mineral surfaces would modify asphaltene adsorption [17]. They found that for kaolinite, asphaltene adsorption decreased from $30 \mathrm{mg} / \mathrm{g}$ (dry clay) to 22 and $13 \mathrm{mg} / \mathrm{g}$ after the pre-addition of 2.3 and $6.6 \%$ water, respectively [17]. Saada et al. [29] concluded that asphaltene adsorption from water-saturated toluene onto the clays kaolinite and illite is related to differences in clay hydrophilicity - the more hydrophilic the clay 
(illite), the lower was asphaltene adsorption. Previously, Menon and Wasan [30] reached a similar conclusion for sodium montmorillonite, for which asphaltene adsorption was reduced significantly on this hydrophilic clay in the presence of pre-adsorbed water. Alipour Tabrizy et al. [31] found that the effects of humidity ('dry' versus 'wet') on asphaltene adsorption were different for calcite, quartz and kaolinite. Adsorption increased by $\sim 60 \%$ for calcite, whereas quartz and kaolinite showed decreases of $23 \%$ and $16 \%$, respectively.

Similar effects were found by Daughney [32] who found that adsorption of crude oil from toluene/heptane mixtures on powdered quartz was reduced in the presence of excess aqueous phases (each with a different $\mathrm{pH}$ ).

In the present paper, our main aim is to determine the role of pre-adsorbed water on asphaltene adsorption on quartz sand, the surface hydration of which is controlled by conditioning to different relative humidity $(\mathrm{RH})$ conditions. Goss determined the adsorption isotherm for water on quartz sand as a function of RH [33]. In a subsequent study [34], the presence of adsorbed water films was shown to reduce the adsorption of small organic molecules from the vapor phase. An "exponential decrease" in adsorption on hydrophilic mineral surfaces was found to occur between 40 and $97 \% \mathrm{RH}$, coinciding with growth of the adsorbed water film [34]. It was therefore of interest to establish whether the adsorption of $n$ heptane-precipitated asphaltene (designated C7A) from toluene solution is affected in a similar way. We also include AFM imaging and NMR wettability measurements on the treated sand to complement the adsorption results. 


\section{Experimental}

\subsection{Materials}

The source of $n$-heptane precipitated asphaltenes (C7A) was a sample of preserved (frozen) oil sands core from the Terre de Grace lease area in Alberta, Canada, kindly supplied by BP Canada. Solvents used ( $n$-heptane, toluene) were AnalaR Grade from Fisher Scientific, UK. Toluene for adsorption experiments was dried to $<5 \mathrm{ppm}$ water (Mettler-Toledo C-20 Coulometric Karl-Fisher titrator) using a PureSolv Micro solvent purification system (Inert Technology Inc. MA, USA) which employs dry nitrogen to push toluene at room temperature through a 304 type stainless steel column containing alumina. The quartz sand used for the adsorption experiments, herein referred to as "ambient sand", was from Sigma-Aldrich, UK. This product was identified for study on the basis of its similar size distribution (particle size 50-70\#, mean particle diameter $\sim 250 \mu \mathrm{m}$ ) as the original core (see later), but without potential interference from surface contamination from bitumen components remaining from cleaning procedures. BET surface area analysis was made using a Belsorp-Mini II (Bel Japan Inc.) using nitrogen as the sorption gas. The sample was degassed using a Belprep II at $150{ }^{\circ} \mathrm{C}$ for 8 hours, with negligible moisture being removed. The salts used to generate the $\mathrm{RH}$ environments from saturated aqueous solutions, $\left(\mathrm{NH}_{4}\right)_{2} \mathrm{SO}_{4}, \mathrm{Mg}\left(\mathrm{NO}_{3}\right)_{2}$, and $\mathrm{MgCl}_{2}$, were obtained from Sigma-Aldrich, UK.

\subsection{Methods}

\subsubsection{Preparation of $\mathrm{n}$-heptane-precipitated asphaltene}


Initially, samples of freshly thawed core were dried for several days to constant weight at a moderate temperature $\left(60^{\circ} \mathrm{C}\right)$ to remove water. Bitumen was then extracted from a weighed sample by Soxhlet extraction using toluene as solvent. Once extraction was complete and the toluene ran clear from the Soxhlet thimble, the apparatus was disassembled, the thimble removed and the small amount of solvent remaining was removed in a stream of dry nitrogen. The particle size distribution of the solids was then determined by micro-sieve analysis. The bitumen solution was filtered (Whatman \#1 filter paper) into a glass beaker, and after the majority of the solvent had been removed by evaporation, approximately 40 volumes of $n$ heptane added to the remaining toluene/bitumen mixture, which was stirred thoroughly for $\sim 12 \mathrm{~h}$ to allow complete destabilization of the asphaltenes (C7A). The mixture was then vacuum-filtered (Whatman \#1 filter paper) to produce solid C7A and the corresponding maltene fraction $(\mathrm{C} 7 \mathrm{M})$, which was recovered after rotary evaporation of the solvent. The C7A was then re-dissolved in toluene and centrifuged (at 10,000 rpm) to sediment any toluene-insoluble (mainly inorganic) material, and the supernatant removed for toluene evaporation. Table 1 contains indicative compositional details of the oil sands sample used in this study, with the corresponding particle size distribution indicating the most abundant size fraction of the solid components shown in Fig. 1.

\section{Table 1.}

Indicative composition of the oil sand sample and extracted asphaltenes.

\begin{tabular}{|l|l|}
\hline Component & \%w/w of oil sand* \\
\hline Solids & $87.0 \pm 0.3$ \\
\hline Bitumen & $13.7 \pm 1.0$ \\
(n-Heptane insolubles, C7A) & $(10.9 \pm 0.8)^{* *}$ \\
\hline
\end{tabular}

\footnotetext{
* Analyses based on dry samples. Three replicate determinations were found to be within the indicated limits.

$* * \% \mathrm{w} / \mathrm{w}$ based on bitumen; asphaltene analysis: $\% \mathrm{C}=78.1 ; \% \mathrm{H}=7.97 ; \% \mathrm{~N}=1.07$.
} 


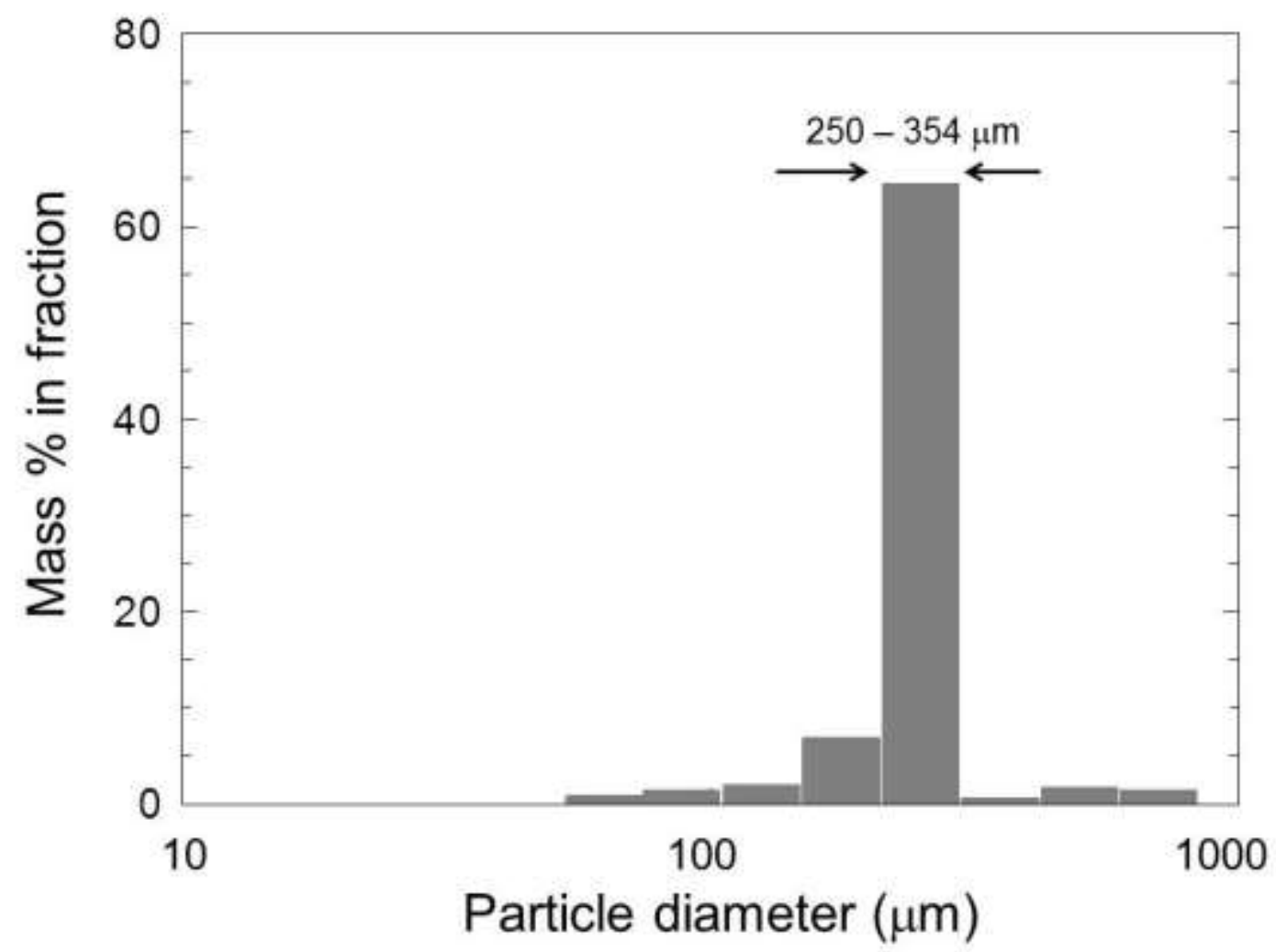

Fig. 1. Particle size distribution from sieve analysis of extracted solids from the BP oil sand sample showing the most abundant (sand) fraction.

\subsubsection{Asphaltene adsorption on sand}

Batch adsorption experiments were carried out by weighing the required masses of the clean commercial sand into $50 \mathrm{~mL}$ polypropylene centrifuge tubes. Initially, an adsorption isotherm was constructed under ambient conditions $\left(22 \pm 2{ }^{\circ} \mathrm{C}\right.$ and $\left.\sim 60 \% \mathrm{RH}\right)$ using untreated clean sand in order to evaluate the form of the isotherm, and typical adsorption levels on this substrate. Appropriate masses (0.5-5 g) of the quartz sand were weighed into the centrifuge tubes and typically $10 \mathrm{~mL}$ of known concentration asphaltene solutions in as-received toluene 
(containing $~ 50 \mathrm{ppm}$ water), prepared on a mass/volume basis was added to each. After capping the tubes, they were equilibrated on a Stuart roller mixer for 24 hours, after which asphaltene depletion in the centrifuged solution (Hettich Lab Technology Rotina 380 centrifuge at 10,000 rpm) was determined by UV-visible spectroscopy (Thermo Scientific Evolution 200) from absorbance measurements at $500 \mathrm{~nm}$ with reference to the original solution. We had established that the concentration-absorbance plot is linear over the working concentration range. Experiments conducted at shorter times indicated that adsorption was complete within the indicated equilibration period. The adsorbed amount of asphaltene $(\Gamma$ in $\mathrm{mg} / \mathrm{g}$ ) was then determined from the equation

$\Gamma=\frac{\left(A_{0}-A\right)}{A_{0} m_{s}} \cdot \frac{C_{0} V}{1000}$

where $A_{0}$ and $A$ are the initial and final absorbances at the measurement wavelength, $C_{0}$ is the original asphaltene concentration in $\mathrm{mg} / \mathrm{L}$ and $V$ is the volume (in $\mathrm{mL}$ ) of the asphaltene solution equilibrated with $m_{s} \mathrm{~g}$ of sand.

The experimental procedure was modified for determining adsorption under the different $\mathrm{RH}$ conditions. In these cases, pre-weighed aliquots of sand were equilibrated for four weeks at $22 \pm 2{ }^{\circ} \mathrm{C}$ in the atmosphere above saturated $\mathrm{RH}$ solutions contained in sealed 2-L glass jars. Table 2 shows the salts used and the corresponding $\mathrm{RH}$ values of their saturated solutions. Prior to the adsorption experiments, the asphaltene solutions were also placed in the corresponding RH atmospheres and left overnight. Nominally 'dry' sand (i.e. $0 \%$ RH) was a sample maintained in an oven at $130{ }^{\circ} \mathrm{C}$ for four weeks and placed together with its corresponding asphaltene solution in a desiccator containing silica gel desiccant 12 hours before the start of the experiments. The sand was not subjected to higher temperatures to 
avoid any changes to the sand structure. Water concentrations in the toluene solutions following the pre-equilibration processes were not determined.

The adsorption experiments involved rapidly adding each asphaltene solution to the respective vials containing the pre-equilibrated sand and immediately capping the centrifuge tube. The tubes were then rolled for the same equilibration period as before, after which the solutions were separated and analyzed. In these cases, the sand was retained for further analysis.

\section{Table 2.}

Relative humidity values of the saturated salt solutions used in this study. Values are interpolated to $22.5^{\circ} \mathrm{C}$ from data given by Greenspan at $20^{\circ}$ and $25^{\circ} \mathrm{C}$ [35].

\begin{tabular}{l|l}
\hline Salt & $\mathrm{RH}(\%)$ \\
\hline None (water) & 100 \\
$\left(\mathrm{NH}_{4}\right)_{2} \mathrm{SO}_{4}$ & $81.2 \pm 0.3$ \\
$\mathrm{Mg}\left(\mathrm{NO}_{3}\right)_{2}$ & $53.6 \pm 0.2$ \\
$\mathrm{MgCl}_{2}$ & $32.9 \pm 0.2$ \\
None (dry sample) & 0 \\
\hline
\end{tabular}

\subsubsection{Low-field NMR wettability measurements}

We were interested to assess the extent to which asphaltene adsorption had changed the wettability of each sand sample, and to this end we used a low-field NMR approach to quantify the distribution of water between the bulk phase and sand surface, as described elsewhere [36].

Aliquots $(0.3 \mathrm{~g})$ of sand removed at the end of the adsorption experiments and dried, were placed in separate $8 \mathrm{~mm}$ NMR tubes, and deionized water $(0.1 \mathrm{~g})$ was added to each. The 
addition of this quantity of water ensures that the sand remains under-saturated, in order to maximize the proportion of bound (surface) water compared to unbound (free) water. Untreated ambient sand (i.e. sand before adsorption and expected to be naturally hydrophilic) was also used for comparison.

Transverse relaxation time $\left(T_{2}\right)$ measurements were then made at ambient temperature using a $20 \mathrm{MHz}$ bench-top NMR magnet (Oxford Instruments, UK) controlled by a Kea-2 spectrometer (Magritek, New Zealand). A CPMG pulse sequence [37,38] was used to measure $T_{2}$ relaxation times at regular intervals using the acquisition parameters given in Table 3. The acquired datasets were then inverted using an 'inverse Laplace transform' (ILT) algorithm in order to generate the $T_{2}$ distributions. The ILT Matlab code was provided by Victoria University (Wellington, New Zealand) [39].

\section{Table 3.}

Acquisition parameters for CPMG pulse sequence.

\begin{tabular}{l|l}
\hline Parameter & Value \\
\hline Number of echoes & 256 \\
Echo time & $20 \mathrm{~ms}$ \\
Number of scans & 64 \\
$\mathrm{P}_{90}$ & $6 \mu \mathrm{s}($ at $-6.6 \mathrm{~dB})$ \\
$\mathrm{P}_{180}$ & $6 \mu \mathrm{s}($ at $-3.3 \mathrm{~dB})$ \\
Dwell time & $1 \mu \mathrm{s}$ \\
Number of echo points & 16 \\
\hline
\end{tabular}




\subsubsection{Atomic force microscopy (AFM) imaging}

AFM is a technique used to produce high-resolution surface images which has been used widely in the study of asphaltenes, e.g. [40-42]. In this study, the surfaces of sand grains taken from post-adsorption at $0 \%$ and $81 \%$ RH were imaged using a Brüker diInnova instrument (Brüker Corp. Santa Barbara, CA, USA) in tapping mode. A clean, ambient sand grain was included for comparison. As is usual, the raw AFM images were imported into Brüker's NanoScope software for further processing.

\section{Results}

\subsection{C7A adsorption isotherm on quartz sand}

In the adsorption experiments described herein we have deliberately maintained relatively low asphaltene concentrations, i.e. at or below the critical nanoaggregate concentration [26], in order to avoid involvement from larger aggregates (clusters). For concentrations below the Langmuir plateau region, the predominant species in toluene solution will be individual asphaltene molecules or dimers [1]. Acevedo et al. [43] report that aggregation of asphaltenes in toluene occurs at $\sim 50 \mathrm{mg} / \mathrm{L}$ and that the formation and adsorption of aggregates on the sand surface might occur at higher concentrations.

Fig. 2 shows the adsorption isotherm for the C7A asphaltene on as-received Sigma-Aldrich quartz sand under ambient conditions. For the asphaltene concentration range studied, the data are satisfactorily fitted to the Langmuir isotherm,

$$
\Gamma=\frac{\Gamma_{\max } C K_{L}}{\left(1+C K_{L}\right)}
$$


where $\Gamma_{\max }$ is the adsorption capacity corresponding to the maximum (Langmuirian plateau) C7A adsorbed amount $(0.203 \mathrm{mg} / \mathrm{g}$ used in this case $), K_{L}$ is the Langmuir constant $(0.142$ $\mathrm{L} / \mathrm{mg}$ ), which is a measure of the affinity of the binding sites for C7A, and as $C$ is the bulk equilibrium asphaltene concentration.

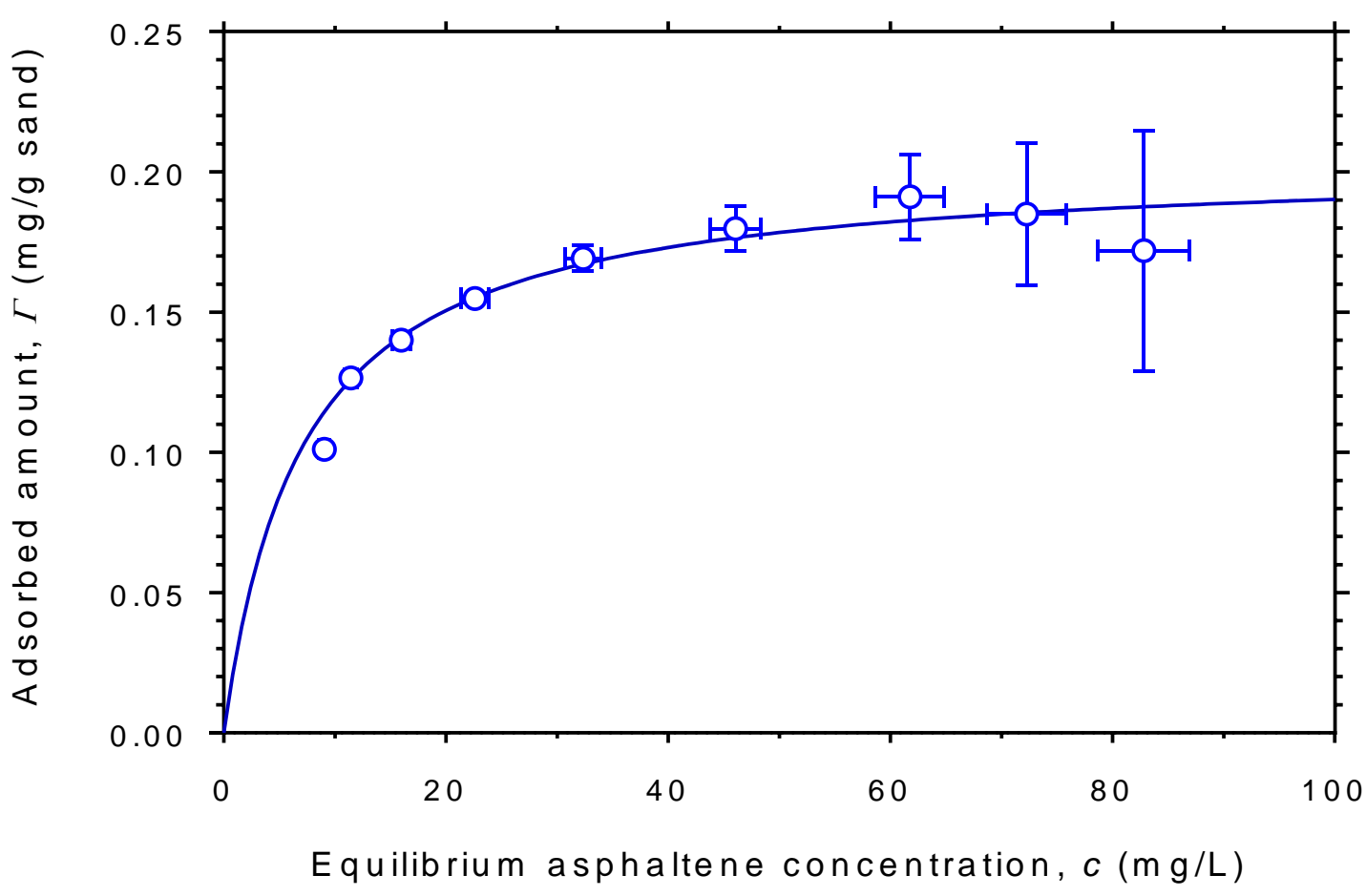

Fig. 2. Low concentration adsorption isotherm of C7A asphaltene on ambient Sigma-Aldrich quartz sand. The line is fitted to the Langmuir isotherm (Eq. 2).

The adsorption capacity is expected to be low for this substrate, owing to the relatively large grain size and absence of significant micro- or meso-porosity. This consequently results in a small specific surface area. A calculation based on smooth spherical particles with a mean diameter of $250 \mu \mathrm{m}$ and bulk density of $2.65 \mathrm{~g} / \mathrm{cm}^{3}$ produces an estimate of $\sim 0.01 \mathrm{~m}^{2} / \mathrm{g}$, which is close to a literature measured value for similarly-sourced sand of $0.03 \mathrm{~m}^{2} / \mathrm{g}$ [44]. Our own attempts to determine the very low BET surface area of this material yielded a value 
of $0.09 \pm 0.02 \mathrm{~m}^{2} / \mathrm{g}$. Higher BET surface areas for other commercial sand samples from the same supplier have been reported for a sieved $(<200 \mu \mathrm{m})$ size fraction $\left(0.34 \mathrm{~m}^{2} / \mathrm{g}\right)$ [33], and for an unspecified particle size $\left(0.65 \mathrm{~m}^{2} / \mathrm{g}\right)$ [31], although the latter is more consistent with a Sigma-Aldrich product with a 1-5 $\mu$ m diameter range.

Therefore, by using the specific surface area for our sand fraction of $0.09 \mathrm{~m}^{2} / \mathrm{g}$ the plateau adsorption capacity (from Fig. 2) corresponds to $\sim 2.2 \mathrm{mg} / \mathrm{m}^{2}$. In Adams' survey, literature adsorption capacity values for quartz/silica were in the range $1-7 \mathrm{mg} / \mathrm{m}^{2}$ depending on the asphaltene and mineral sources as well as the method of determination [1].

By assuming an asphaltene molecular mass of 750 [26], the plateau adsorption capacity of $\sim 2.2 \mathrm{mg} / \mathrm{m}^{2}$ corresponds to a low average adsorbed area of $\sim 57 \AA^{2} /$ molecule. However, assuming the same asphaltene density $\left(1200 \mathrm{~kg} / \mathrm{m}^{3}\right)$ as used by Adams [1], indicates an adsorbed layer thickness of $\sim 1.8 \mathrm{~nm}$. Thus, these dimensions are more consistent with adsorption of nanoaggregates which, according to the Yen-Mullins model have sizes in the range of $\sim 2-5 \mathrm{~nm}[1,26]$, rather than single molecules.

\subsection{Effect of RH on C7A adsorption}

Initial asphaltene concentrations in the range $135-140 \mathrm{mg} / \mathrm{L}$ were selected for the $\mathrm{RH}$ experiments to allow depletion through adsorption to result in bulk concentrations which were approximately comparable with the Langmuir plateau region in Fig. 2 determined for ambient sand. Thus, in Fig. 3 are shown duplicate C7A asphaltene adsorption data as a function of RH. These results show that asphaltene adsorption decreases approximately fourfold as RH increases between 0 and $100 \%$, which spans the highest vale region shown in Fig. 
2. The decrease over the RH range is not uniform, however, but indicates a region, between $\sim 40-80 \% \mathrm{RH}$, in which adsorption remains near-constant at $\sim 0.3 \mathrm{mg} / \mathrm{g}$.

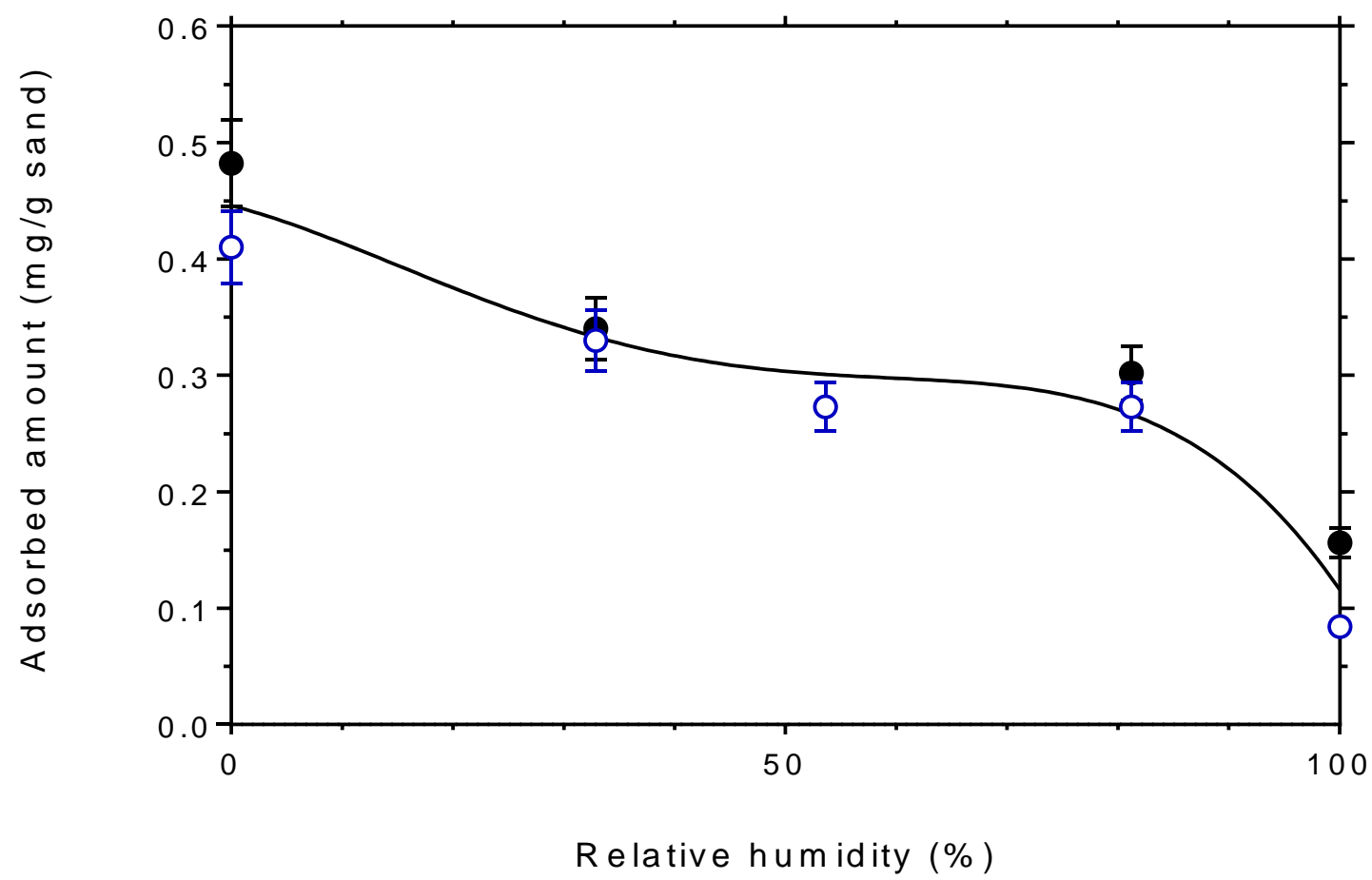

Fig. 3. Plot showing adsorbed amounts of asphaltene on quartz sand as a function of RH for two different sets of experiments. The drawn line is to guide the eye.

\subsection{NMR wettability measurements}

Proton $T_{2}$ NMR relaxation measurements are sensitive to local proton environments. In aqueous systems, the relaxation spectra enable the distribution of water protons in different environments to be determined; these range from mobile bulk water, exhibiting long relaxation times, to more restricted conditions, such as bound surface water, with shorter relaxation times. In the present study, hydrophobic (oil-wet) surfaces with little affinity for water would be expected to exhibit longer $T_{2}$ relaxation times. Conversely, water-wet 
hydrophilic surfaces would be expected to produce shorter relaxation times. Thus, by measuring relaxation spectra over time, fluid-solid interactions can be monitored, thereby enabling wettability or wettability changes to be inferred [36].

In Fig. 4 are shown $T_{2}$ relaxation spectra for deionized water in contact with sand after asphaltene adsorption at $0 \%$ and $100 \% \mathrm{RH}$ for illustrative purposes, together with the corresponding results for clean sand.

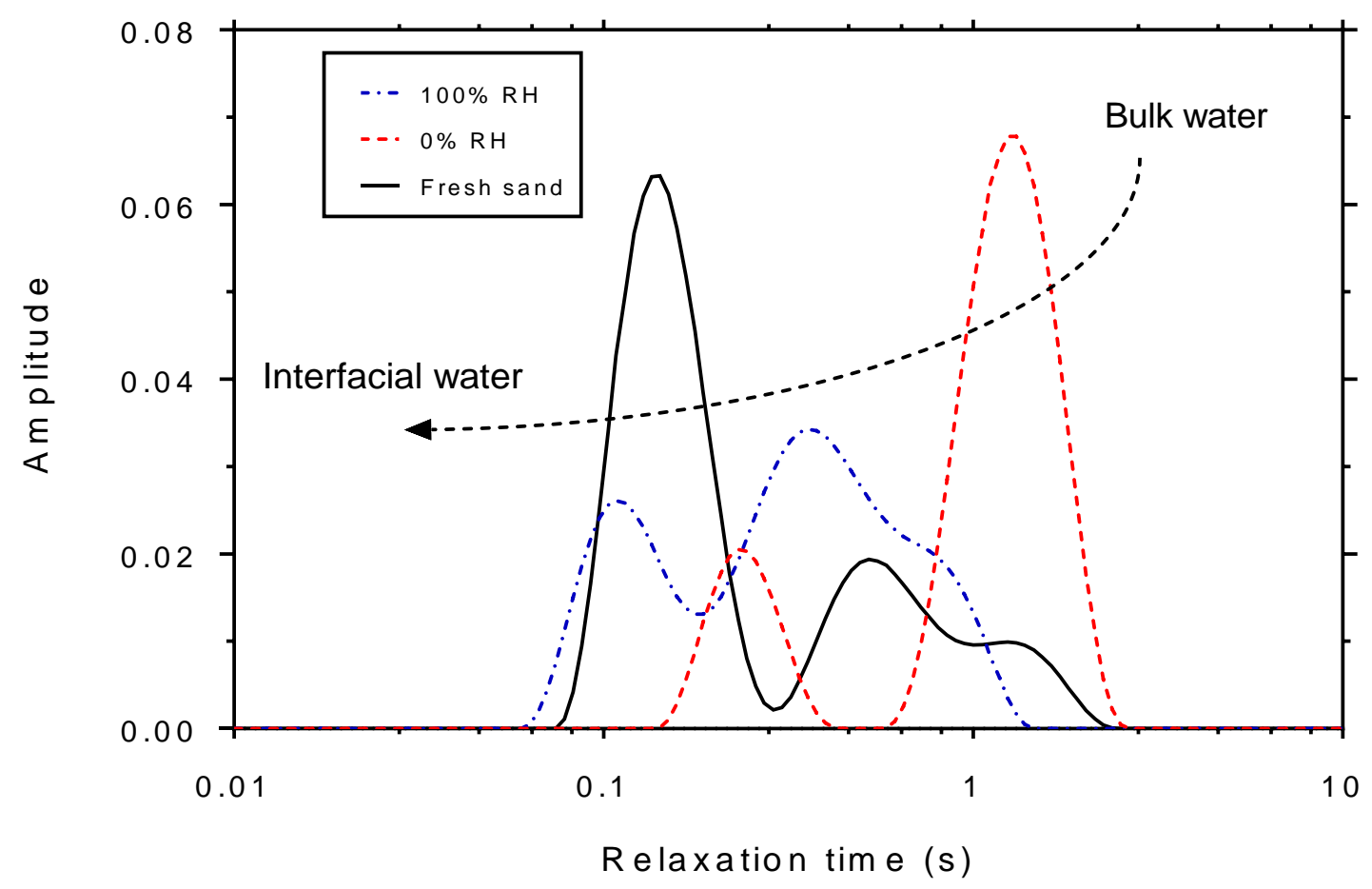

Fig. 4. $T_{2}$ relaxation spectra for deionized water in contact with asphaltene-coated sand produced at $0 \%$ RH (dashed line) and 100\% RH (dot-dashed line). For comparison, deionized water in contact with fresh sand is also shown (continuous line).

It can be seen that for the hydrophilic fresh sand, the relaxation spectrum contains a large peak at $\sim 0.1 \mathrm{~s}$, consistent with surface-bound water. Excess bulk water is responsible for the longer relaxation time components. In contrast to this, the sand sample that had been subjected to asphaltene adsorption under 0\% RH conditions shows a much smaller short 
relaxation time component, with the majority of the response comprising a longer relaxation time, typical of a predominantly hydrophobic sand sample [36]. For the sand produced at $100 \% \mathrm{RH}$, however, the spectrum is seen to indicate mixed wettability characteristics.

The complexity of these spectra is often reduced by calculating the geometric mean $T_{2}$ values ( $\left.T_{2 g m}\right)$ using Eq. 3, where $A_{i}$ is the amplitude of each $T_{2 i}$ value, i.e. [36]

$$
T_{2 g m}=\exp \left(\frac{\sum_{1}^{n} A_{i} \ln T_{2 i}}{\sum_{1}^{n} A_{i}}\right)
$$

Thus, in Fig. 5 are the resultant plots of $T_{2 g m}$ against time for sand obtained under four of the five RH adsorption conditions, together with an untreated ambient sand sample. For each asphaltene-treated sand it is seen that $T_{2 g m}$ decreases with time, in contrast with the fresh ambient sand, which is time-invariant. For the $100 \% \mathrm{RH}$ sand sample, $T_{2 g m}$ is seen to decrease very rapidly to a value which is close to that found for ambient sand. This is consistent with comparatively low asphaltene adsorption causing the surface to become weakly hydrophobic, as evidenced by a high initial $T_{2 g m}$ value, but which then easily reverts back to its natural hydrophilic state when contacted by water.

On the other hand, for sand samples which have undergone asphaltene adsorption at $0 \%, 33 \%$ and $81 \% \mathrm{RH}$, the original state of the sand surface is less-easily recovered, as shown by $T_{2 g m}$ decreasing more gradually with time. This is consistent with the adsorbed asphaltene layer being thicker or more firmly bound to the surface, thereby preventing its displacement and presenting a stronger hydrophobic barrier against the added water contacting the sand surface. The $T_{2 g m}$ time dependence is very similar for these three RH values, although the initial $T_{2 g m}$ values are different, and consistent with the amounts of adsorbed asphaltene (Fig. $3)$. 


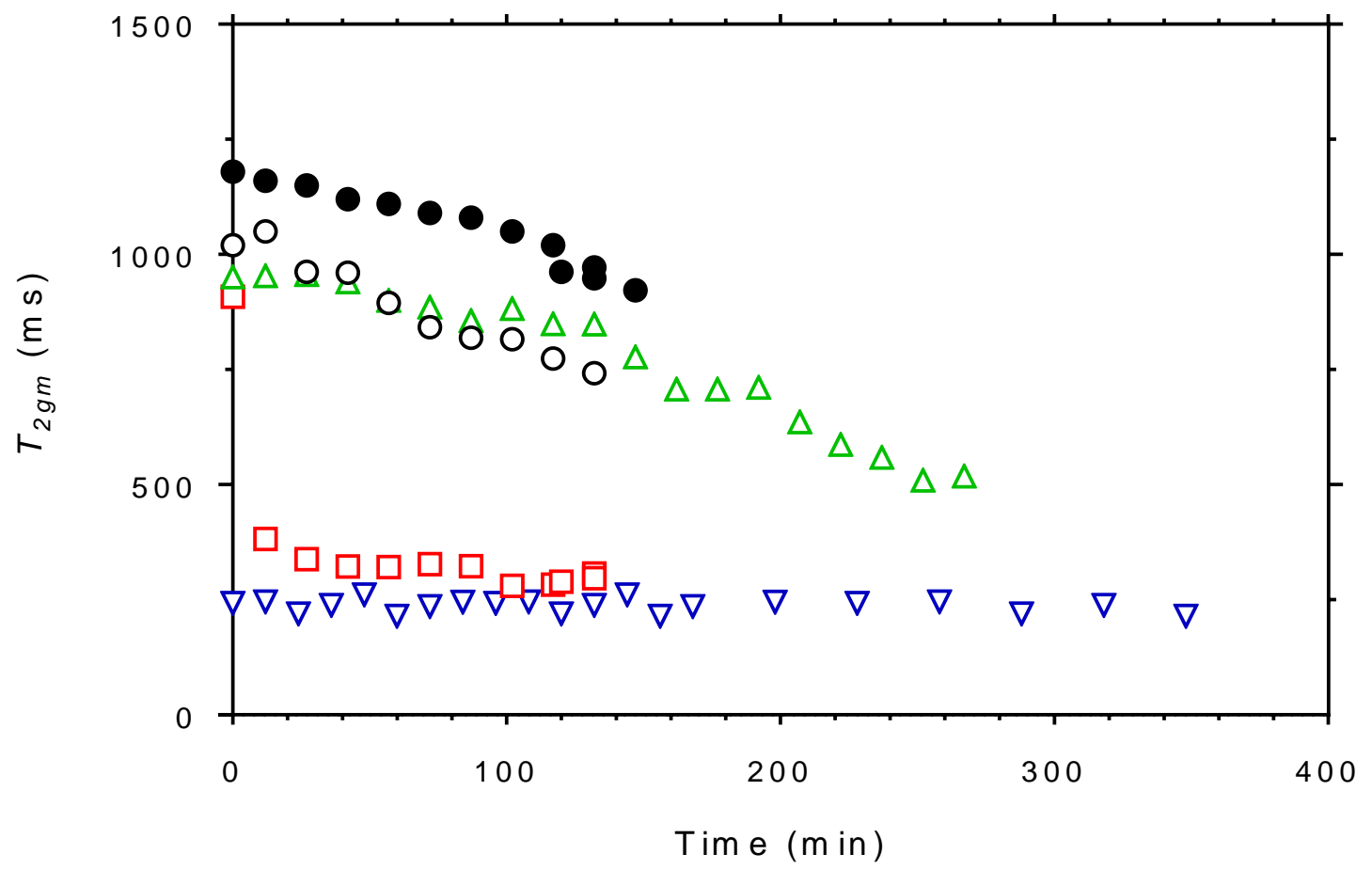

Fig. 5. Plots of $T_{2 g m}$ against time for deionized water in contact with asphaltene-coated sand: 0\% RH (filled circles); 32.8\% RH (open circles); $81.0 \%$ RH (open triangles); and 100\% RH (open squares). Data are also shown for untreated sand (open inverted triangles).

\subsection{AFM analysis}

AFM has been used to confirm differences in asphaltene adsorption at two contrasting RH conditions $(0 \%$ and $81 \%)$. Images were obtained for sand grains recovered from the adsorption experiments. These were compared with an untreated, ambient sand grain. Thus, Fig. 6 shows the respective images, each representing an area of $0.5 \mu \mathrm{m} \times 0.5 \mu \mathrm{m}$, which shows that a thicker, more uniform and possibly smoother layer is formed on sand at $0 \% \mathrm{RH}$, compared with that produced at $81 \% \mathrm{RH}$. 
At this scale, the thickness of the layers are $\sim 6 \mathrm{~nm}$ for asphaltene adsorbed at $0 \% \mathrm{RH}$ and $\sim 4 \mathrm{~nm}$ at $81 \% \mathrm{RH}$, compared with a baseline value of $\sim 0.5 \mathrm{~nm}$ for untreated sand. The adsorbed layer at $0 \% \mathrm{RH}$ is therefore directionally as expected based on the adsorption data. Unsurprisingly, images produced over larger areas (e.g. $1 \mu \mathrm{m} \times 1 \mu \mathrm{m}$ and $3 \mu \mathrm{m} \times 3 \mu \mathrm{m}$ ) are subject to a greater influence from the uneven sand grain surfaces and are therefore not shown.
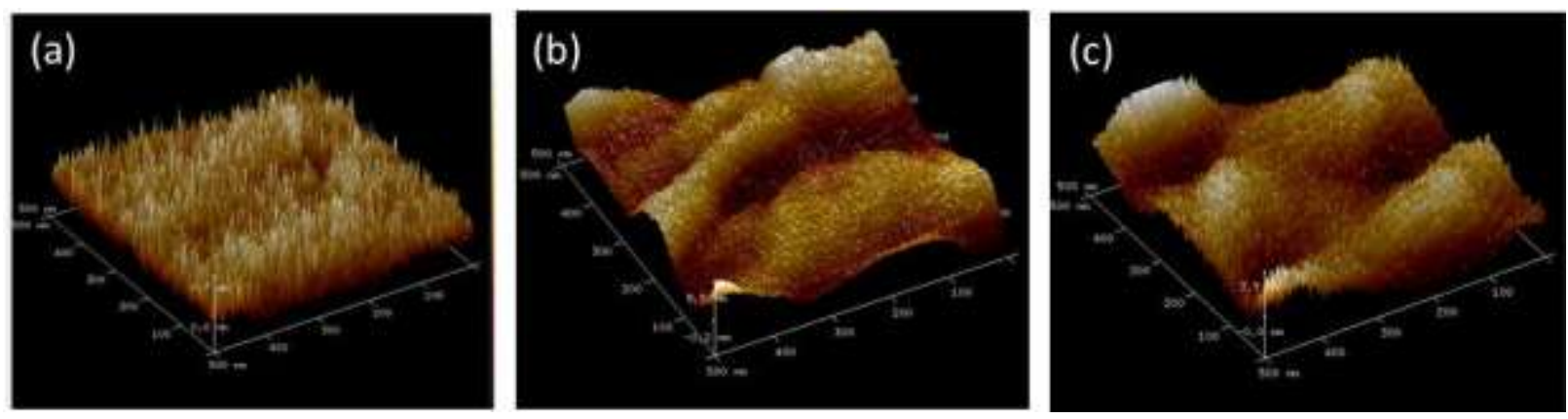

Fig. 6. $0.5 \mu \mathrm{m} \times 0.5 \mu \mathrm{m}$ AFM images for (a) plain sand, and sand with asphaltene adsorbed at (b) $0 \% \mathrm{RH}$, and (c) $81 \% \mathrm{RH}$. The measurements were all made under ambient conditions using a set point of $2 \mathrm{~V}, 256$ samples/lines, and a scan rate of $4 \mathrm{~Hz}$.

\section{Discussion}

In order to compare asphaltene adsorption with corresponding literature water adsorption isotherm data for a similar sand sample [33], results from Fig. 3 are re-plotted in Fig. 7 on a molar basis. Whilst accepting that the two sand samples are not likely to be identical, it is apparent that the two isotherms are fundamentally complementary, such that when water adsorption is high, asphaltene adsorption is low, and vice versa (but note the different $y$-axis scales). 


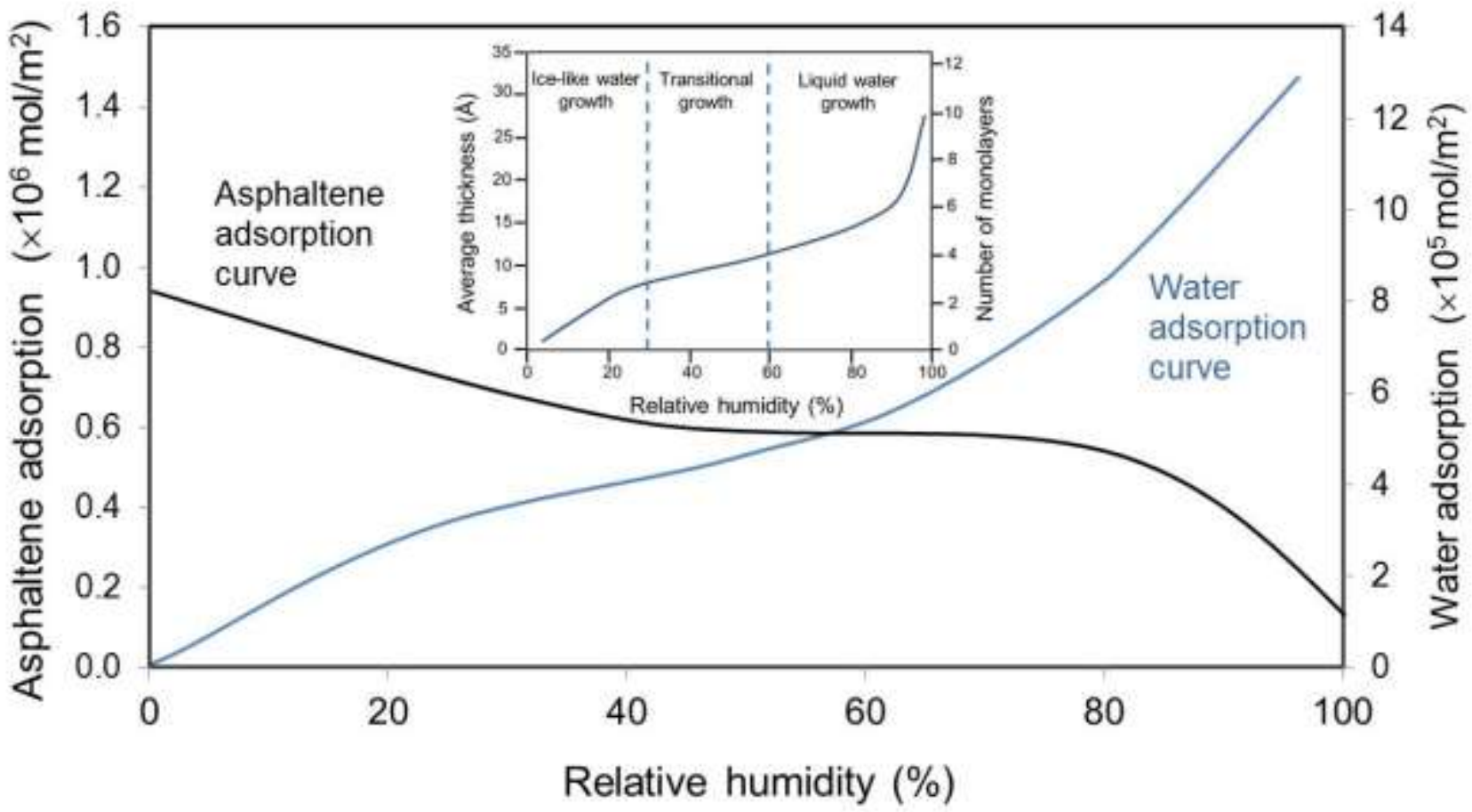

Fig. 7. Comparison between asphaltene (left axis) and water vapor (right axis) adsorption on quartz sand as a function of RH. The asphaltene adsorption curve is obtained from the data in Fig. 3 and a specific surface area of $0.09 \mathrm{~m}^{2} / \mathrm{g}$ (see text), whereas the water adsorption curve is derived from [33]. The insert shows further data from Asay and Kim [45] relating the thickness of the adsorbed water layers on silica to RH and defines three water growth regions referred to in the text.

Our results indicate that asphaltene adsorption is very dependent on the hydrated state of the sand surface. At $0 \% \mathrm{RH}$, the silica surface will contain siloxane groups and silanol groups containing H-bonded hydroxyl groups, and free hydroxyl groups, either isolated or geminal [46]. In general, the main adsorption sites are considered to be the surface silanol hydroxyls and these are normally available for H-bonding as well as $\pi$-bonding with aromatic species [47]. In the absence of water, the nanoaggregate surface concentration is $0.45 \mathrm{mg} / \mathrm{g}\left(\sim 1 \times 10^{-6}\right.$ $\mathrm{mol} / \mathrm{m}^{2}$ from Figs. 3 and 7) and this decreases as the RH increases, which suggests that water 
competes for the surface sites. Water adsorption increases relatively rapidly up to $\sim 30 \% \mathrm{RH}$ [33], and thereafter asphaltene adsorption is seen to be relatively constant as far as $\sim 80 \% \mathrm{RH}$. In this range, water adsorption continues to increase, albeit more slowly until $\sim 60 \% \mathrm{RH}$. Above this RH value, however, water multilayers begin to form rapidly [33] at which stage asphaltene adsorption decreases, although, as also observed previously by Collins and Melrose for adsorption on kaolinite, it is not prevented completely [17]. As shown in Fig. 7 (insert), three regions were identified and described by Asay and Kim [45] as ice-like growth in the low $\mathrm{RH}$ range, which corresponds to a build-up of $\sim 3$ monolayers (with a thickness of $\sim 6 \AA$ ), followed by a transition region with the addition of a further monolayer in the mid-RH range, and finally liquid water (multilayer) growth at high RH.

As we show in Fig. 8, the remarkably linear dependence of asphaltene adsorption on the thickness of the adsorbed water film is very evident. Here, we have plotted our asphaltene adsorption data as a function of the thickness of the adsorbed water layer taken from Asay and $\operatorname{Kim}[45]$. 


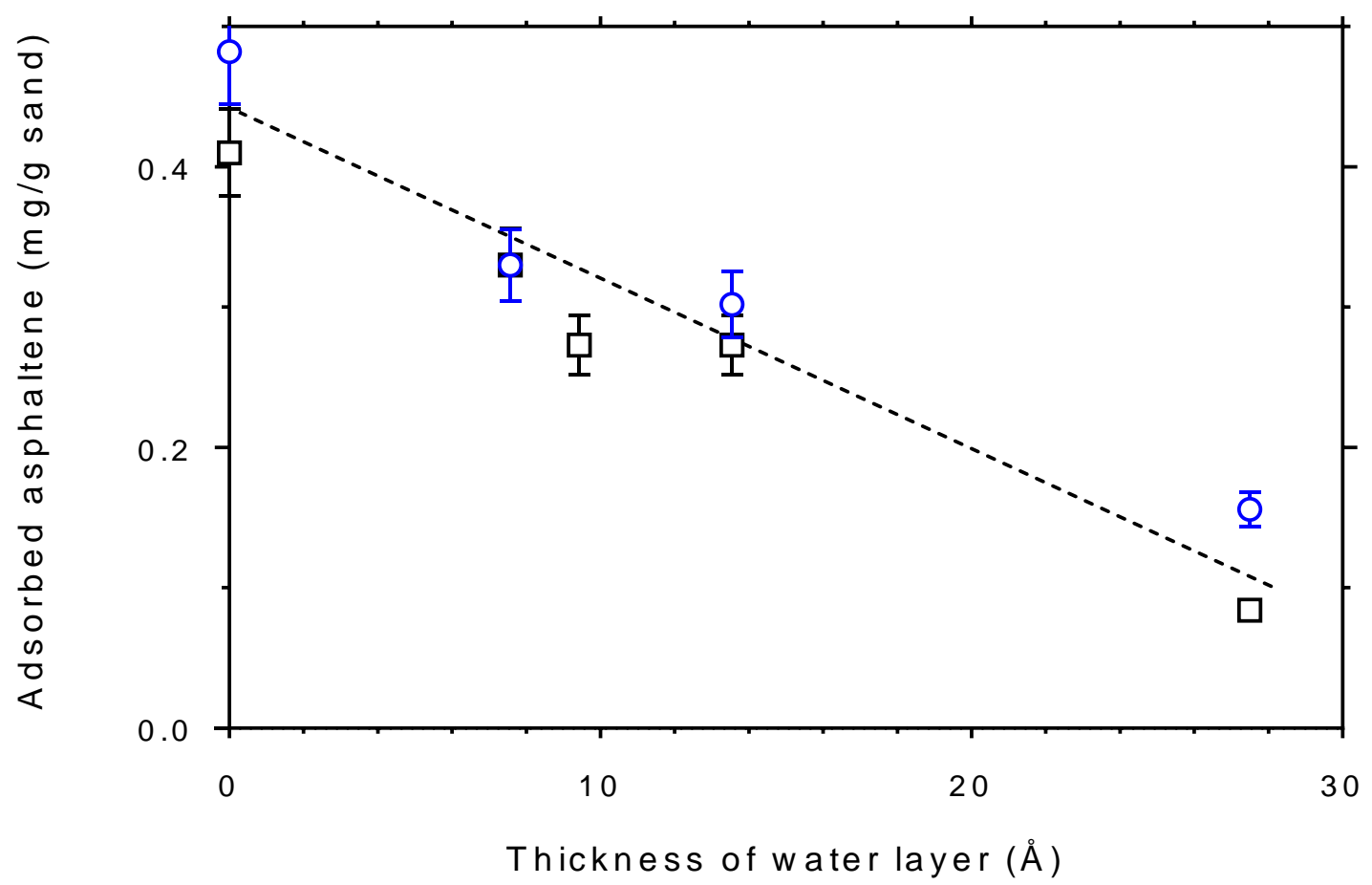

Fig. 8. Plot showing the effect of the thickness of the adsorbed water layer on asphaltene adsorption. The asphaltene adsorption data are from the present study, and water thickness data are from Asay and Kim [45]. The dashed line is the least squares fit to the data $\left(\mathrm{R}^{2}=\right.$ $0.920)$.

In the mid-range RH region, where liquid water growth occurs on the silica surface [45], the asphaltene surface concentration is $\sim 0.6 \times 10^{-6} \mathrm{~mol} / \mathrm{m}^{2}$. This is almost double that of the lowest surface hydration condition, which will contain more adsorption (silanol) sites. The effect of this will be effectively to reduce the area per nanoaggregate and increase the density of the adsorbed layer when compared with the presence of surface water. The increased thicknesses shown in the AFM images suggest that some further aggregation may also occur on the dehydrated sand surface. The thickness based on the AFM images shown in Fig. 6 is found to be consistent with the trend seen in the adsorption experiments. 
In the 'dry' $(0 \% \mathrm{RH})$ condition, the surface will contain the maximum number of available silanol adsorption sites. Asphaltene adsorption can therefore potentially undergo hydrogen and $\pi$-bonding to the silanol groups through hetero-groups and aromatic centers, respectively. However, an additional adsorption mode at the silica-toluene interface, most appropriately operative in the absence of water, has been reported as arising through electric field effects [48]. It was proposed that observations of increased surface precipitation of dispersed asphaltenes at a silica surface are explained by electrostatic attraction [48]. Asphaltenes dispersed in non-polar media are known to be electrically charged [49-54], and depending on the method of preparation and the type and concentration of solvent, electrodeposition experiments, in particular, have shown that asphaltene particles carry either positive or negative charges $[52,54]$. However, in pure solvents, and specifically when indigenous resins or added surface-active species are absent, dispersed asphaltenes (which will comprise clusters and larger aggregates derived from nanoaggregates) are positively charged $[53,55]$.

In contrast to this, the charge on bare acidic silica surfaces in non-aqueous solvents is almost exclusively negative as a result of dissociation of surface silanol groups [56,57]. Thus, without offering a definitive explanation here, it is possible that electrical effects may contribute to the adsorption behavior, particularly in the absence of water.

However, as the RH increases to $\sim 30 \%$, adsorbed water will become increasingly involved in hydrogen bonding with the surface silanol groups resulting in the $\mathrm{H}$-bonded ice-like structure [45]. Water will therefore compete with the asphaltene for $\mathrm{H}$-bonding sites and reduce the electrostatic contributions to adsorption through charge dissipation. It is seen from Fig. 7 that within the ice-like and transitional growth regions, asphaltene adsorption remains approximately constant. 
Upon exceeding $\sim 60 \% \mathrm{RH}$, however, the influence of the silica surface becomes further reduced due to adsorption occurring at an increasingly water-like interface, where the adsorbed water film increases in thickness beyond 5-10 molecular layers at 90\% RH [34].

We summarize the foregoing with reference to the schematic representation of the hydrated silica surface shown in Fig. 9. Here, we show the relationship between asphaltene adsorption and the three water growth regions of Asay and Kim. From this analysis it is apparent that any effects due to water structure can only be subtle, with the water layer thickness dictating asphaltene adsorption. 


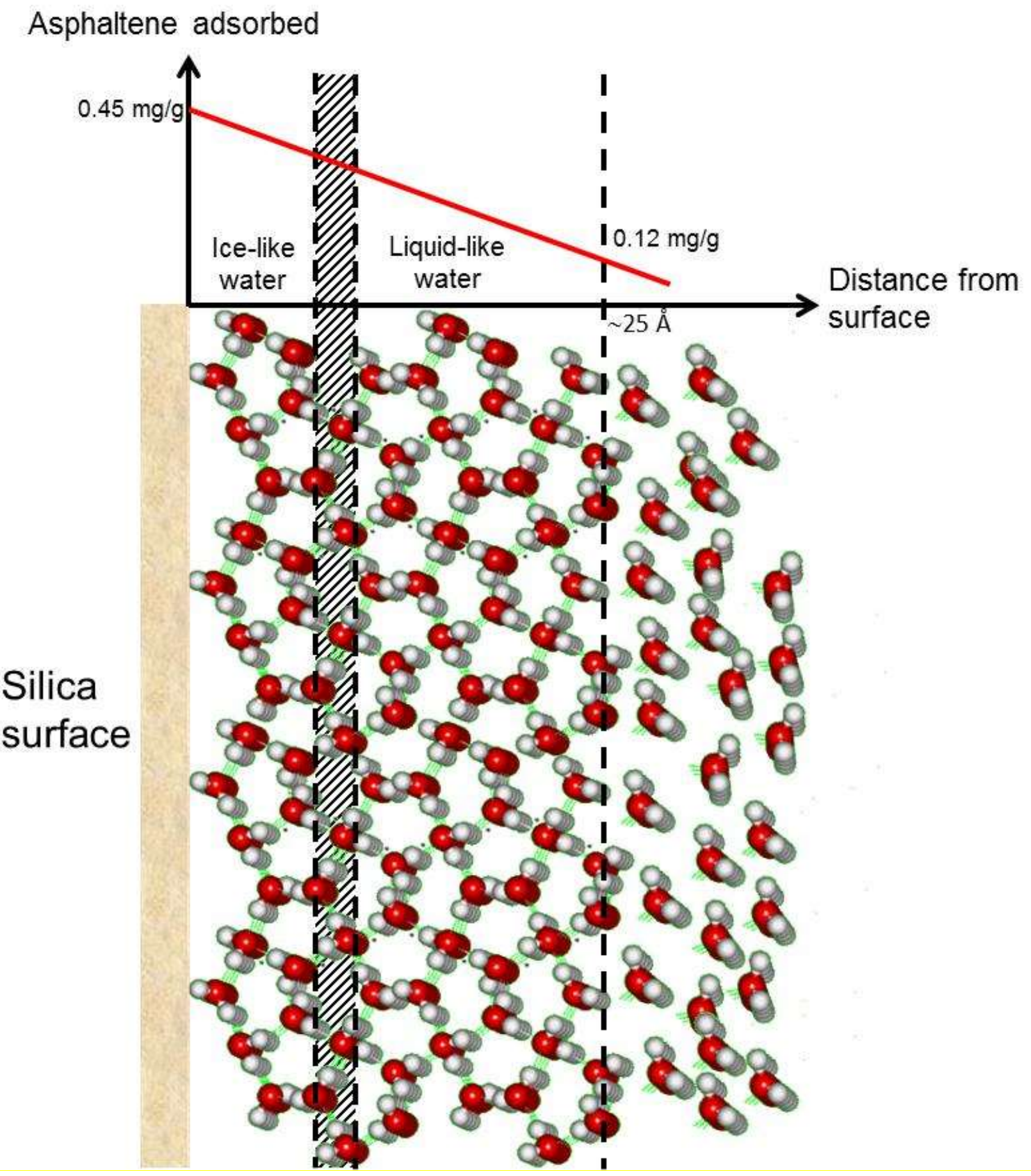

Fig. 9. Schematic representation of the adsorbed water structure profile at a silica surface and its relation to the present $\mathrm{RH}$ asphaltene adsorption data. The water structure regions are from ref. [45], with the hatched area representing the transitional growth region. 
Since the asphaltene adsorption is strongly influenced by the presence of pre-adsorbed water, we were also interested to determine whether there is a consequent effect on sand wettability, and to this end, we have used water proton $T_{2}$ relaxation as a measure. As described above, the $T_{2}$ relaxation spectrum reflects the different water environments in the sample, and in particular distinguishes interfacial (short $T_{2}$ ) from bulk water (longer $T_{2}$ ). Thus, hydrophilic surfaces with a comparatively high water affinity will have a shorter relaxation time compared with hydrophobic surfaces. Asphaltene coatings on surfaces are expected to be hydrophobic, and characterized by higher relaxation times, which we present as geometric mean $T_{2 g m}$ values to reflect the complete spectrum of relaxation times. Thus, Fig. 5 shows that for all the samples tested, $T_{2 g m}$ decreases with time indicating that the surfaces become more hydrophilic as water penetrates (and/or displaces [58]) the adsorbed asphaltene film [36]. For the $100 \% \mathrm{RH}$ sample, which has the lowest asphaltene adsorption, $T_{2 g m}$ is seen to decrease within minutes, almost reaching the baseline value for ambient sand. However, for the other samples, the decrease in $T_{2 g m}$ occurred over a period of hours, consistent with the presence of thicker or possibly more complete adsorbed asphaltene layers on these samples.

\section{Conclusions and implications}

There is considerable interest in quartz sand (silica) surfaces, particularly in the oil industry, where this mineral is the geological basis for a large proportion of oil reservoirs. Indeed, oil sands make up a significant proportion of global unconventional heavy oil and bitumen reserves, especially in Canada. Understanding silica/water/oil interfacial chemistry is an important aspect in improving recovery techniques, and the role of water is one of its key influences. 
The aim of the present work was to investigate previous observations in the literature which suggested that the presence of water reduces asphaltene adsorption on different mineral surfaces. Thus, by controlling the RH environments to which the sand was exposed, we have demonstrated a direct link between water and asphaltene adsorption, possibly for the first time. We have identified complementarity between the two isotherms, such that the highest asphaltene adsorption is observed in the absence of a water film, and this decreases linearly as the thickness of the adsorbed surface water increases (with increasing $\mathrm{RH}$ ). Not only does this suggest the possibility that asphaltene and water compete for the same (silanol) surface sites, but the adsorbed water may present a barrier to asphaltene adsorption, as the silica surface structure of the silica changes due to hydration [45]. In the absence of water, formation of thicker adsorbed films may also be influenced by electric field effects as described recently by Acevedo et al. [48].

The present findings are in general agreement with extensive work carried out by Goss and coworkers in which the presence of water films on hydrophilic mineral surfaces were shown to inhibit adsorption of a range of organic compounds from the vapor-phase $[33,34]$. The discontinuity of the RH dependence of sorption (at $\sim 26 \% \mathrm{RH}$ ) was considered to be due to incomplete coverage of the quartz surface $(<\sim 26 \%)$, leaving some sites unoccupied, or by competition with water molecules that are preferentially adsorbed [33]. Our results have also demonstrated, possibly for the first time, similar effects in a liquid-phase adsorption process.

AFM imaging has provided further evidence (Fig. 6) that the adsorbed asphaltene layer is thicker $(\sim 6 \mathrm{~nm})$ on the $0 \% \mathrm{RH}$ sample compared with the $81 \%$ sample $(\sim 4 \mathrm{~nm})$. Even allowing for the non-ideal nature of the sand grain surface, these adsorbed layer thicknesses are consistent with values obtained on more ideal planar surfaces, summarized by Adams [1]. 
Low-field NMR has provided additional evidence for asphaltene adsorption on sand by means of $T_{2}$ relaxation time wettability measurements. The increased thickness of the asphaltene film is indicated by higher initial $T_{2}$ values, and reinforced by slower rates of change of $T_{2 g m}$. The rapid decrease of $T_{2 g m}$ with time for the $100 \% \mathrm{RH}$ condition is also consistent with the lowest adsorbed amount of asphaltene.

The present results could add support for the existence of water films separating bitumen from solid surfaces in natural oil sands [16-19], and may also serve to explain differences between asphaltene adsorption data reported in the literature. Finally, the experimental approach adopted here could be used to complement recent modeling studies on the adsorption of oil components in the presence of water, e.g. $[59,60]$.

\section{Acknowledgements}

We are grateful to Allan Peats of BP Canada, for supplying the oil sands core used to derive the $n$-heptane-precipitated asphaltene and sand particle size distribution, and to BP America for establishing the Centre for Petroleum and Surface Chemistry and the award of a $\mathrm{PhD}$ studentship to VG. We appreciate the valuable contributions to the experimental work made by Aristeidis Tsiamis (ambient RH adsorption data), Rifat Shaikh (AFM) and Richard Fields (BET analysis), while Dr. Huang Zeng (BP America) is thanked for his support, interest and valuable discussions throughout the course of our work on asphaltene chemistry. Finally, we are grateful to reviewers who raised several constructive and interesting points which greatly improved the paper. 


\section{References}

[1] J.J. Adams, Energy Fuels 28 (2014) 2831-2856.

[2] D.M. Grist, E.L. Neustadter, K.P. Whittingham, J. Can. Pet. Tech. 20 (1981) 74-78.

[3] R.A. Mohammed, A.I. Bailey, P.F. Luckham, S.E. Taylor, Colloids Surf. A 80 (1993) 223-235.

[4] C. Dicharry, D. Arla, A. Sinquin, A. Graciaa, P. Bouriat, J. Colloid Interface Sci. 297 (2006) 785-791.

[5] J. Czarnecki, P. Tchoukov, T. Dabros, Z. Xu, Can. J. Chem. Eng. 91 (2013) 13651371.

[6] V. Pauchard, J.P. Rane, S. Banerjee, Langmuir 30 (2014) 12795-12803.

[7] S.E. Taylor, Chem. Ind. (20) (1992) 770-773.

[8] E.M. Freer, C.J. Radke, J. Adhesion, 80 (2004) 481-496.

[9] M. Sedghi, L. Goual, W. Welch, J. Kubelka, J. Phys. Chem. B 117 (2013) 5765-5776.

[10] X. Tan, H. Fenniri, M.R. Gray, Energy Fuels 23 (2009) 3687-3693.

[11] M.R. Gray, R.R. Tykwinski, J.M. Stryker, X. Tan, Energy Fuels 25 (2011) 31253134.

[12] L.M. da Costa, S.R. Stoyanov, S. Gusarov, X. Tan, M.R. Gray, J.M. Stryker, R. Tykwinski, J. W. de M. Carneiro, P.R. Seidl, A. Kovalenko, Energy Fuels 26 (2012) 2727-2735.

[13] J. Murgich, D. Merino-Garcia, S.I. Andersen, J.M. del Rio, C.L. Galeana, Langmuir 18 (2002) 9080-9086.

[14] S.I. Andersen, J.M. del Rio, D. Khvostitchenko, S.Shakir, C. Lira-Galeana, Langmuir, 17 (2001) 307-313.

[15] D.S. Khvostichenko, S.I. Andersen, Energy Fuels 22 (2008) 3096-3103.

[16] K. Takamura, Can. J. Chem. Eng. 60 (1982) 538-545.

[17] S.H. Collins, J.C. Melrose, SPE J. 23 (1983) 249-258.

[18] J. Masliyah, Z. Zhou, Z. Xu, J. Czarnecki, H. Hamza, Can. J. Chem. Eng. 82 (2004) 628-654.

[19] J. Czarnecki, B. Radoev, L.L. Schramm, R. Slavchev, Adv. Colloid Interface Sci. 114-115 (2005) 53-60. 
[20] S.T. Dubey, M.H. Waxman, SPE Res. Eng. (1991) 391-395. doi.org/10.2118/18462PA

[21] M. Chacon-Patino, C. Blanco-Tirado, J. Orrego-Ruiz, A. Gomez-Escudero, Energy Fuels 29 (2015) 1323-1331.

[22] W. Anderson, J. Pet. Tech. 38 (1986) 1125-1144.

[23] S.T. Kim, M.-E. Boudh-Hir. M-E., G.A. Mansoori, Paper SPE 20700 presented at $65^{\text {th }}$ Annual Technical Conference and Exhibition of the Society of Petroleum Engineers, New Orleans, LA, September 23-26, 1990.

[24] C. Drummond, J. Israelachvili, J. Pet. Sci. Eng. 45 (2004) 61-68.

[25] K.L. Gawrys, M. Spiecker, P.K. Kilpatrick, Pet. Sci. Tech. 21 (2003) 461-489.

[26] O.C. Mullins, H. Sabbah, J. Eyssautier, A.E. Pomerantz, L. Barré, A.B. Andrews, Y. Ruiz-Morales, F. Mostowfi, R. McFarlane, L. Goual, R. Lepkowicz, T. Cooper, J. Orbulescu, R.M. Leblanc, J. Edwards, R.N. Zare, Energy Fuels 26 (2012) 3986-4003.

[27] M. Szymula, A.W. Marczewski, Appl. Surface Sci. 196 (2002) 301-311.

[28] A.W. Marczewski, M. Szymula, Colloids Surf. A 208 (2002) 259-266.

[29] A. Saada, B. Siffert, E. Papirer, J. Colloid Interface Sci. 174 (1995)185-190.

[30] V.B. Menon, D.T. Wasan, Colloids Surf. 19 (1986) 107-122.

[31] V. Alipour Tabrizy, R. Denoyel, A.A. Hamouda, Colloids Surf. A 384 (2011) 98108.

[32] C.J. Daughney, Org. Geochem. 31 (2000) 147-158.

[33] K.-U. Goss, Environ. Sci. Technol. 26 (1992) 2267-2294.

[34] K.-U. Goss, R.P. Schwarzenbach, J. Colloid Interface Sci. 252 (2002) 31-41.

[35] L. Greenspan, J. Res. Nat. Bur. Stand. (US) 81 (1977) 89-96.

[36] V. Gonzalez, M. Jones, S.E. Taylor, Energy Fuels 30 (2016) 844-853.

[37] H.Y. Carr, E.M. Purcell, Phys. Rev. 94 (1954) 630-638.

[38] S. Meiboom, D. Gil, Rev. Sci. Instr. 29 (1959) 688-961.

[39] Y.-Q. Song, L. Venkataramanan, M.D. Hurlimann, M. Flaum, P. Frulla, C. Straley, J. Magn. Reson. 154 (2002) 261-268.

[40] A. Hannisdal, M.-H. Ese, P.V. Hemmingsen, J. Sjöblom, Colloids Surf. A 276 (2006) 45-58. 
[41] S. Simon, J. Jestin, T. Palermo, L. Barre, Energy Fuels 23 (2009) 306-313.

[42] L. Goual, A. Abudu, Energy Fuels 24 (2010) 469-474.

[43] S. Acevedo, M.A. Ranaudo, J.C. Pereira, J. Castillo, A. Fernandez, P. Perez, M. Caetano, Fuel 78 (1999) 997.

[44] A. Pitois, L.G. Abrahamsen, P.I. Ivanov, N.D. Bryan, J. Colloid Interface Sci. 325 (2008) 93-100.

[45] D.B. Asay, S.H. Kim, J. Phys. Chem. B 109 (2005) 16760-16763.

[46] M.L. Hair and W. Hertl, J. Phys. Chem. 73 (1969) 4269-4276.

[47] M.L. Hair, J. Non-Cryst. Solids, 19 (1975) 199-209.

[48] S. Acevedo, J. Castillo, E.H. Del Carpio, Energy Fuels 28 (2014) 4905-4910.

[49] S.M. Hashmi, A. Firoozabadi, Soft Matter 7 (2011) 8384-8391.

[50] S.M. Hashmi, A. Firoozabadi, Soft Matter 8 (2012) 1878-1883.

[51] R. Gaikwad, A. Hande, S. Das, S.K. Mitra, T. Thundat, Langmuir 31 (2015) 679-684.

[52] D.S. Khvostichenko, S.I. Andersen, Energy Fuels 23 (2009) 811-819.

[53] D.S. Khvostichenko, S.I. Andersen, Energy Fuels 24 (2010) 2327-2336.

[54] S.E. Taylor, Fuel, 77 (1998) 821-828.

[55] W.Th. Kok, A.J. Tudos, M. Grutters, A.G. Shepherd, Energy Fuels 25 (201) 208-214.

[56] M.E. Labib, R. Williams. J. Colloid Interface Sci. 97 (1984) 356-366.

[57] M.E. Labib, Colloids Surf. 29 (1988) 293-304.

[58] U. Farooq, J. Sjöblom, G. Øye, J. Disp. Sci. Tech. 32 (2011) 1388-1395.

[59] J. Zhong, P. Wang, Y. Zhang, Y. Yan, S. Hu, J. Zhang, Energy 59 (2013) 295-300.

[60] G. Wu, X. Zhu, H. Ji, D. Chen, Chemosphere 119 (2015) 242-249. 